\title{
Importancia etnozoológica de herpetos en bosques de la selva pluvial central del Chocó
}

\section{Ethnozoological Importance of herps in rain forests of central Chocó rainforest}

\section{Eric Yair Cuesta-Ríos ${ }^{1}$, Luis Eladio Rentería Moreno²}

\section{Resumen}

Mediante la combinación de herramientas cualitativas y metodologías participativas que permitieron la interacción entre investigadores y actores de la comunidad, se determinó la importancia etnozoológica de la herpetofauna para los pobladores de los alrededores de la Estación Ambiental Tutunendo de Quibdó, reportando un total de dos clases, cuatro órdenes, 10 familias, 14 géneros y 16 especies de herpetos que son objeto de uso por la comunidad como los Quelonios: Chelydra acutirostris (Bache), Kinosternon leucostomum (Tortuguita), Kinosternon scorpioides (Tapaculo) y Rhynoclemmys nasuta (Tortuga blanca), son las especies más utilizadas. Se registraron seis categorías de uso como (1) Alimentación que involucró 11 especies, (2) uso como mascota con nueve, (3) Medicina tradicional-mágico religioso con ocho, (4) Ornamento con siete, (5) Control por prevención con cinco y (6) Comercio con cuatro especies; las tortugas es el grupo taxonómico más explotado en la comunidad.

Palabras clave: Chocó; Etnozoología; Herpetofauna; Usos.
1. Instituto de Investigaciones Ambientales del Pacífico. «JVN», Grupo Investigación Conocimiento, Manejo y Conservación de los Ecosistemas del Chocó Biogeográfico, Quibdó, Colombia. e-mail: cuestarios@gmail.com

2. Especialista en Gerencia de Recursos Naturales, Docente Investigador del Grupo de Herpetología y Curador Colección Científica de Referencia Zoológica del Chocó-Herpetología de la Universidad Tecnológica del Chocó, Quibdó, Chocó, Colombia.e-mail: lueremo@gmail.com

Recibido: 26 de enero de 2012 Aceptado: 28 de marzo de 2012

\begin{abstract}
By combining qualitative tools and methodologies participatory allowed interaction between researchers and actors community, we determined the importance of the herpetofauna ethnozoological for the residents of the surrounding Tutunendo Environmental Station of Pretoria, reporting a total of two classes, four orders, 10 families, 14 genera and 16 species of herps that are subject to use by the community as Chelonian: Chelydra acutirostris (Bache), Kinosternon leucostomum (Turtle) Kinosternon scorpioides (Tapaculo) and Rhynoclemmys nasuta (Tortuga white), are the most used. There were six categories of use as (1) Food that involved 11 species, (2) use as a pet nine, (3) Traditional Medicine-religious magic eight, (4) Christmas with seven, (5) Control and prevention with five (6) Trade with four species, the turtle is the most exploited taxonomic group in the community
\end{abstract}

Keywords: Chocó; Etnozoología; Herpetofauna; Uses.

\section{Introducción}

Para contar la historia de la relación hombre y herpetofauna es preciso remontarse hasta los inicios de la vida contada desde la narración bíblica cuando Eva fue tentada por la serpiente. Según el texto bíblico tras haber sido creados, Adán y Eva residían en el jardín del Edén en perfecta armonía con Dios, el único mandato al que debían acogerse era la abstención de comer del árbol de la ciencia del bien y del mal, cuyo consumo ocasionaría la muerte. Sin embargo, Eva y por su intermediación Adán, cedieron a la tentación de la serpiente (identificada con Satán) y descubrieron, comiendo del 
árbol, su desnudez. La consecuencia de la violación del mandato los llevó a la muerte y la expulsión del jardín del Edén.

A partir de este momento se formaron eslabones que han trascendido mediante centenares de generaciones por medio de saberes populares o conocimientos tradicionales, producto de espacios y construcciones colectivas, especificas e interdependientes, que se articulan con todos los procesos vitales y culturales de los grupos humanos (Escobar 2007), demostrando que el conocimiento se ha transformado, apareciendo una nueva mirada donde lo científico no es el único modelo teórico-práctico de asumir la verdad y la realidad, porque existen otras realidades, otras verdades, otras formas de conocer, manejar, utilizar, visualizar, percibir y soñar la naturaleza que han permanecido, en espacios no científicos, que se ha transmitido de generación a generación, milenio a milenio, pueblo a pueblo, persona a persona.

Según Escobar (2007), la etnozoología es el área de las ciencias naturales donde más se han desarrollado estas alternativas de conocimiento, ya antes áreas del conocimiento como la etnografía, la etnología, la antropología y la sociología se habían introducido en el estudio de estos pueblos y sus conocimientos asociados. Es así que el prefijo etnos hace referencia a los aspectos humanos y conocimientos específicos de pueblos o etnias, es decir, a los conocimientos de agrupaciones naturales de individuos de igual cultura.

Ahora bien, por ser consultados estos «saberes», generalmente a nivel de intereses específicos o sectoriales de cada disciplina y no sobrepasar más que ciertos aspectos complementarios, la coherencia y racionalidad del «conocimiento tradicional» de sectores o grupos humanos, se puede perder en la segregación que de él se hace, por esta razón con la presente investigación se busca reconocer los usos tradicionales de la herpetofauna y de esta manera contribuir a la recuperación de estos conocimientos y que esto repercute en el uso racional de la fauna y su entorno.

Área de estudio. Esta investigación de corte etnozoológico se realizó en las inmediaciones de La Estación Ambiental Tutunendo (EAT) ubicada en el corregimiento de Tutunendo (municipio de Quibdó, Chocó) a los $5^{\circ} 48^{\prime} 00^{\prime \prime}$ norte y $76^{\circ} 31^{\prime}$ 00 " oeste, con características predominantes de zona de vida selva pluvial central. Según Poveda-M et al. (2004), ambientalmente corresponde a la zona de temperatura megatermal $\left(25,7^{\circ} \mathrm{C}\right.$ a $\left.27,9^{\circ} \mathrm{C}\right)$, de precipitación pluvial muy alta $(8494$ a $13670 \mathrm{~mm})$ y de balance hídrico perhúmedo a superhúmedo (248,9 a 385,8).

El corregimiento de Tutunendo posee una población de 3.500 habitantes aproximadamente, compuesta principalmente por afrocolombianos $(90 \%)$, indígenas y colonos. La agricultura y la explotación maderera son las actividades económicas principales y en segundo orden la minería, la pesca y la caza.

\section{Materiales y métodos}

El levantamiento de la información fue abordada mediante el método etnográfico, combinando herramientas cualitativas y metodologías participativas que permitieron una interacción entre los investigadores y los actores de la comunidad, con el propósito de conocer cómo los pobladores realizan sus actividades cotidianas con respecto al uso de la herpetofauna, de tal manera que se realizaron observaciones directas en campo y encuestas semiestructuradas, utilizando un formato general de encuestas adaptado de Mosquera (2001), Moreno y Toral (2001) y Escobedo y Ríos (2003) que incluía preguntas sobre uso, conocimiento y percepción del recurso en la zona y respaldados en la determinación taxonómica in-situ con base en el conocimiento de los informantes claves y confirmadas mediante las guías de campo de Renjifo y Lundberg (1999) y Páez et al. (2002), para anfibios y reptiles.

\section{Resultados y discusión}

Registramos 16 especies de herpetos presente en las inmediaciones de la EAT aprovechada por los habitantes de esta zona, que se agrupan en 14 géneros, 10 familias y cuatro órdenes (Anura, Testudinata, Crocodilia y Squamata) (Tabla 1). Este listado incluye cuatro de la 24 especies reportadas por Moya-Robledo (2006) y 11 de las 39 registradas por Renteria-M et al. (2007), en sus estudios herpetológicos para la misma zona.

De manera particular se evidenció que las especies que presentan un mayor rango o tipos de usos son C. serpentina (bache), K. spurelli (tortuguita), K. scorpiodes (tapaculo) y $R$. nasuta (tortuga blanca), las cuales son utilizadas tanto como alimentación, como objeto de comercio, mascotas, con fin mágico religioso y uso ornamental (Figura 1). Lo anterior muestra el importante papel que juegan los quelonios para las comunidades del Pacífico, donde este grupo taxonómico es muy explotado, a pesar que sus especies sean el grupo de vertebrados más amenazados de Colombia.

De esta manera la herpetofauna se constituye en un recurso fundamental dentro de las manifestaciones socioeconómicas y culturales de esta región, convirtiendo a estas especies en vulnerables a la extinción local, por las constantes amenazas que son notorias en los últimos tiempos debido a la expansión agrícola, la minería y la tala selectiva que se ha convertido en una de las actividades socioeconómicas más importantes de la región (Cuesta-Ríos y Valencia-Mazo 2006).

Utilización del recurso. De acuerdo con los resultados se obtuvo un total de seis categorías de uso, que son en orden de importancia para la comunidad: médico-tradicional y mágico-religioso, alimentación, mascota, control por prevención 


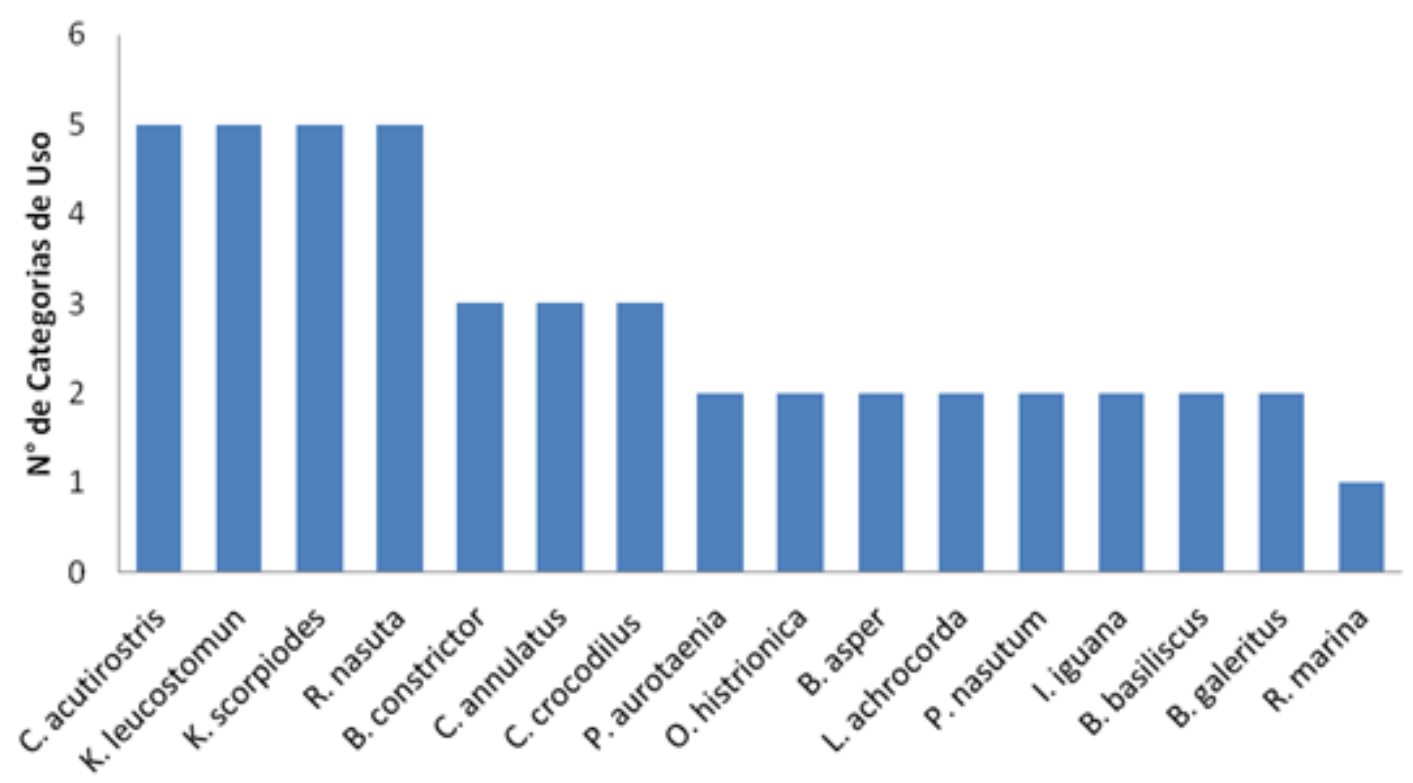

Figura 1. Especies de herpetos de mayor uso por los pobladores en los alrededores de la Estación Ambiental Tutunendo (EAT), Quibdó, Chocó, Colombia

Tabla 1

Composición taxonómica de la herpetofauna utilizada por los pobladores en los alrededores de la Estación Ambiental Tutunendo (EAT), Quibdó, Chocó, Colombia

\begin{tabular}{|c|c|c|c|}
\hline Clase & Orden & Familia & Especies \\
\hline \multirow{3}{*}{ Amphibia } & Anura & Dendrobatidae & Oophaga histriónica \\
\hline & & & Phyllobates aurotaenia \\
\hline & & Bufonidae & Rhynella marina \\
\hline \multirow{13}{*}{ Reptilia } & Testudinae & Chelydridae & Chelydra acutirostris \\
\hline & & Kinosternidae & Kinosternon leucostomun \\
\hline & & & Kinosternon scorpiodes \\
\hline & & Geoemydidae & Rhinoclemmys nasuta \\
\hline & Squamata & Viperidae & Bothrops asper \\
\hline & & & Lachesis achrocorda \\
\hline & & & Porthidium nasutum \\
\hline & & Iguanidae & Iguana iguana \\
\hline & & Corytophanidae & Basiliscus basiliscus \\
\hline & & & Basiliscus galeritus \\
\hline & & Boidae & Boa constrictor \\
\hline & & & Corallus annulatus \\
\hline & Crocodylia & Alligatoridae & Caiman crocodilus \\
\hline
\end{tabular}


y en menor grado de importancia el uso ornamental y comercio (Tabla 2).

El uso de la herpetofauna depende notablemente de la caza, que para la zona se puede considerar según los argumentos de Cuesta-Ríos et al. (2007), como una actividad socioeconómica secundaria que articula los sistemas productivos, social y ecológicos, la cual se ha adquirido a través de una larga cadena de generaciones, una serie de conocimientos, que permiten adaptar sus técnicas al aprovechamiento de estos recursos tanto a través de proceso de difusión como por experiencia propia a lo largo de una carrera continua de ensayo y error, y cuyo objetivo principal es la obtención de proteína animal, aunque sin dejar de lado otros usos, los cuales aportan ingresos económicos y algún bienestar a las familias. Concordando con Ulloa et al. (1996), quienes sustentan que las presas de caza no solo aportan alimento, suelen ser importantes para las familias como fuentes medicinales, simbólicas (mediante prestigios, adivinación, premonición, protección entre otras), rituales, elaboración de objetos, mascota, en intercambio comercial y uso social (creación de alianzas y parentescos).
Médico-tradicional y mágico-religioso. La categoría médico-tradicional y mágico-religiosa, informa 13 especies (P. aurotaenia, O. histrionica, $R$. marina, C. acutirostris, $K$. leucostomun, K. scorpiodes, R. nasuta, B. asper, L. achrocorda, P. nasutum, B. constrictor, C. annulatus, C. crocodilus) de las cuales cinco (R. marina, B. asper, $L$. achrocorda, $P$. nasutum y Caiman crocodilus), presentan propiedades medicinales con características antiofídicas, antiasmática, analgésica, cicatrizante y dermatológicas y nueve [P. aurotaenia, O. histrionica, B. asper, L. achrocorda, $P$. nasutum (Guerrero y Ballesteros 2006) C. acutirostris, K. leucostomun, $K$. scorpiodes, $R$. nasuta] están asociadas con un sinnúmero de creencias, mitos y leyendas, como hechizos, maleficios, que por respeto a los médicos tradicionales o curanderos no se describen en el escrito y la adquisición de destrezas animales, por ejemplo, poder contener la respiración por mucho tiempo debajo del agua, esto en el caso de ser consumido el corazón de las tortugas listadas en el presente documento. Según), son tradiciones estrechamente arraigadas con las comunidades afrocolombianas del Chocó, en especial en la población adulta quienes explican aconteci-

Tabla 2

Usos de la herpetofauna en las inmediaciones de la Estación Ambiental Tutunendo

\begin{tabular}{|c|c|c|c|c|c|c|c|c|}
\hline \multirow[t]{2}{*}{ Clase } & \multirow[t]{2}{*}{ Especie } & \multirow[t]{2}{*}{ Nombre vulgar } & \multicolumn{6}{|c|}{ Usos } \\
\hline & & & $\mathbf{A L}$ & CM & MS & M-R & OR & $\mathbf{C P}$ \\
\hline & Phyllobates aurotaenia & Rana venenosa & & & & $x$ & $x$ & \\
\hline \multirow[t]{8}{*}{ Amphibia } & Oophaga histrionica & Rana venenosa & & & & $x$ & $x$ & \\
\hline & Rhynella marina & Sapo & & & & & & \\
\hline & Chelydra acutirostris & Tortuga bache & $x$ & $x$ & $x$ & $x$ & $x$ & \\
\hline & Kinosternon leucostomun & Tortuguita & $x$ & $x$ & $x$ & $x$ & $x$ & \\
\hline & Kinosternon scorpiodes & Tortuga tapaculo & $x$ & $x$ & $x$ & $x$ & $x$ & \\
\hline & Rhinoclemmys nasuta & Tortuga blanca & $x$ & $x$ & $x$ & $x$ & $x$ & \\
\hline & Bothrops asper & Equis & & & & $x$ & & $x$ \\
\hline & Lachesis achrocorda & Verrugoso & & & & $x$ & & $x$ \\
\hline \multirow[t]{7}{*}{ Reptilia } & Porthidium nasutum & Equis 24 & & & & $x$ & & $x$ \\
\hline & Iguana iguana & Iguana & $x$ & & $x$ & & & \\
\hline & Basiliscus basiliscus & Chochora & $x$ & & $x$ & & & \\
\hline & Basiliscus galeritus & Chochora & $x$ & & $x$ & & & \\
\hline & Boa constrictor & Boa, Jepa & & & $x$ & $x$ & & $x$ \\
\hline & Corallus annulatus & Jepa & & & $x$ & $x$ & & $x$ \\
\hline & Caiman crocodilus & Babilla & $x$ & & & $x$ & $x$ & \\
\hline Total & & & 8 & 4 & 9 & 13 & 7 & 5 \\
\hline
\end{tabular}

$(\mathrm{AL})$ : Alimentación (CM): Comercio (MS): Mascota $(\mathrm{OR})$ : Ornamento (M-R): Mágico-Religioso; $(\mathrm{CP})$ Control por prevención 


\section{Bioetnia Volumen 9 № 2 (julio-diciembre), 2012}

mientos naturales y sobre-naturales a través de los animales, siendo la palabra un tesoro que merece respeto.

El uso de anfibios y reptiles con fines médico-tradicionales o etnomédicos por esta comunidad se puede considerar como una manifestación del efecto parcial de las deficiencias en el sector salud dirigida a los pobladores de esta región, que hacen de la medicina tradicional una fuente de segundas oportunidades de dignidad y vida, poniendo en evidencia la constante interacción entre los habitantes locales y su medio natural, concepto ratificado por Rivas et al. (2003), donde manifiesta que las comunidades del Chocó han utilizado de generación en generación órganos y productos derivados de algunas especies de animales para satisfacer necesidades medicinales que por condiciones de aislamiento o económicas no son satisfechas por la medicina científica.

En relación con la utilidad mágico-religiosa el uso de animales juega un papel preponderante en las dogmas y cultura de esta comunidad porque algunos de estos animales poseen o se les atribuyan propiedades animadas, destrezas y beneficios que no tienen los fármacos ni las plantas, propiedades que se trasladan al hombre y que además de tener beneficio a la salud, son más tenidas en cuenta en el concepto mágico religioso porque incluyen oraciones, fe e invocación de espíritus.

A pesar de la riqueza herpetofaunística, la influencia de las doctrinas religiosas sobre toda católicas, ponen en un alto grado de vulnerabilidad las poblaciones de ofidios debido al rechazo y repudio colectivo de las serpientes, pues alrededor de estas se han generado más mitos que con el resto de especies de anfibios y reptiles ya que estas se les asocia con el mal. Además se profesa que si una mujer en estado de gestación o en período menstrual va al monte es perseguida por el animal, ya que su humor en ese estado es fuerte y si es mordida en estado de gestación muere al instante. De igual forma se dice que no se puede ir a cazar mal dormido (tener relaciones sexuales en la madrugada) por que puede ser perseguido por serpientes.

Por otra parte se cree que con un caldo preparado con carne de las víboras B. asper (equis), L. muta (verrugoso) y $P$. nasuta (equis veinticuatro), se pude curar el dolor intenso de muelas, aun que esta práctica no es muy común porque existe el temor a «condenarse» al consumir un animal asociado con el mal según los mitos católicos.

En relación con el grupo de los Anuros sobre todo las ranas venenosas, se dice que sus huesos son empleados en prácticas rituales para elaborar conjuros, para causarles mal a otras personas.

Alimentación. En investigaciones de corte etnozoológico, la caza para la obtención de proteína animal para la alimentación del cazador y su familia es uno de los usos más importantes dentro de las comunidades rurales, pero el consumo de reptiles en esta región no es común, aunque se destaca la utilización de ocho especies como las tortugas $C$. serpentina (bache), K. spurelli (tortuguita), K. scorpiodes (tapaculo) y $R$. nasuta (tortuga blanca), los lagartos $B$. basiliscus, $B$. galeritus (chochoras) y el consumo de la carne de C. crocodilus (babilla), que es considerada una de las carnes más sanas y que además posee una exquisitez y consistencia que no tienen el resto de las carnes mencionadas antes. Ojasti (1993), afirma que el mayor aporte socioeconómico actual de la fauna silvestre neotropical es su contribución nutricional a las comunidades rurales; en las selvas tropicales en la mayoría de los casos cubre el requerimiento mínimo de proteínas de la población indígena y aporta un promedio de $20 \%$ de la demanda proteica de dichas poblaciones, que para esta zona solo aporta valores que oscilan entre $1 \%$ y $4 \%$ de la proteína animal que la gente consume.

Mascotas. Nueve especies de herpetos son utilizados en la comunidad de Tutunendo como mascotas; esta categoría de uso para este grupo taxonómico es frecuente en la región, siendo su motivación principal la adquisición de reptiles como las tortugas. Es muy frecuente ver a los niños de la comunidad en constante interacción con lagartos como $I$. iguana, B. basiliscus y B. galeritus. Esta categoría se puede considerar como un uso no consuntivo, pero es importante porque hace parte de las tradiciones culturales y recreacionales de los pobladores.

Ornamentos. La fabricación de ornamentos con órganos, sustancias o partes de herpetos está limitada a siete especies; los pobladores de la localidad utilizan las pieles de la babilla (C. crocodilus) para adornar sus viviendas, también es importante resaltar la importancia en la fabricación de bodoques que recobra el grupo de las ranas venenosas $P$. aurotaenia y O. histrionicus, con las sustancias tóxicas exudadas por su piel, que es usado para la caza de aves y mamíferos que posan en las copas de los arbóreas, también es rescatable la utilización de los caparazones de las tortugas para adornos y para el depósito de sal y algunas verduras.

Control por precaución. Entre los usos registramos el control por precaución, donde la mayor parte de los animales implicados son las serpientes. Esta actividad es ocasional en donde los pobladores sacrifican cruelmente animales, como las serpientes consideradas malignas y peligrosas y también sapos (R. marina) que según los pobladores, la orina y la leche de este animal causa inflamaciones en la parte del cuerpo donde se aplica. Lugo (2005), argumenta que algunos animales causan daños en las áreas de cultivo, por tanto los habitantes los identifican como un conflicto de uso presente y consideran algunos animales como plagas en sus cultivos, por lo que los sacrifican para el control y cuidado de linderos de cultivo.

Cabe resaltar que aunque para este estudio solo se registran dos especies de serpientes en esta categoría, esta debería incluir todo el grupo de ofidios, porque según Rentería y 
Rivas (2009), quienes estudiaron la etnozoología de la ofidiofauna asociada con cultivos agrícolas en esta localidad, los pobladores sacrifican a todas las serpientes que se encuentran, sin discriminar si es venenosa o no, pues según sus creencias todas las serpientes son peligrosas (a excepción de la platanillo Leptophis ahaetulla).

Esta categoría fue descrita por Ojasti (2000), quien argumenta que fuera del marco legal, el control es un pretexto frecuente para matar por capricho o ignorancia diversos animales, sobre todo carnívoros y serpientes, que carecen de atributos cinegéticos. Muchos autores manifiestan que las comunidades negras e indígenas han mantenido una relación armónica con la fauna pero debido a la cultura de esta comunidad las serpientes son maléficas y por consiguiente hay que controlarlas; existe otro grupo de animales que son controlados debido a que (como argumentan los pobladores) son un peligro para su seguridad.

Comercio. La poca demanda que tiene el mercado de anfibios y reptiles, convierten a esta categoría en una actividad insipiente; sin embargo, en ocasiones comercializan con turistas y visitantes, animales vivos como las tortugas y pieles (principalmente de babilla), todo con el fin de obtener recursos económicos para suplir otras necesidades, el dinero obtenido es utilizado para comprar otros productos que complementan y balancean la dieta familiar. Bodmer y Pezo (1999), mencionan que la fauna silvestre constituye un recurso importante en la economía regional ya sea como fuente alimenticia o venta de mercado.

En comunidades de estas características, los grupos humanos, en especial las etnias, no aíslan sus conocimientos, no lo parcelan ni sectorizan, todo hace parte de un todo, de una cosmovisión integral del mundo, no presentan una visión unilateral o bidimensional del mundo, es una cosmovisión multidimensional que une el pasado con el futuro, lo inerte con lo vivo, lo material con lo espiritual, enlazan normas, valores, naturaleza, costumbres, la salud, la enfermedad y muchos otros conceptos, con lo mágico, las leyendas, los mitos que son parte indisoluble de sus conocimientos y prácticas cotidianas.

\section{Conclusión}

Las particularidades etnográficas, socioeconómicas, culturales y ambientales hacen que exista una amplia tradición del uso de la herpetofauna en la zona ya que el reconocimiento y uso de estos animales hacen parte de la cotidianidad de la gente, siendo la cacería de subsistencia la actividad que más relevancia toma.

Según los análisis del estudio, las especies de anuros $P$. aurotaenia y $O$. histrionicus, los ofidios y los quelonios, son los grupos de herpetos más vulnerables a la extinción local, debido a su explotación y a las constantes amenazas que implican la expansión agrícola, la minería y la tala selectiva que se ha convertido en una de las actividades socioeconómicas más importantes de la región.

\section{Literatura citada}

Bodmer, R.E., E. Pezo. 1999. Análisis económico de la venta de carne de monte y exportación de pieles en Loreto, Perú. En: T. Fang, O. Montenegro, R. Bodmer (eds.). Manejo y conservación de fauna silvestre en América Latina. La Paz: Instituto de Ecología. pp. 169-170.

Cuesta-Ríos, E., J. Valencia-Mazo. 2006. Patrones de uso tradicional de la fauna nativa en el área de influencia de la Estación Ambiental Tutunendo (EAT) Quibdó, Chocó, Colombia. Trabajo de grado. Universidad Tecnológica del Chocó. Facultad de Ciencias Básicas. Programa de Biología con Énfasis en Recursos Naturales. Quibdó. 98 pp.

Cuesta-Ríos, E., J. Valencia-Mazo, A. M. Jiménez-Ortega. 2007. Aprovechamiento de los vertebrados terrestres por una comunidad humana en los bosques tropicales (Tutunendo, Chocó, Colombia). Revista Institucional Universidad Tecnológica del Chocó 26 (2): 37-43.

Escobar-Berón, G. 2007. Etnobiología: introducción al paradigma de la etnobiología. Etnociencias y yagé. [en línea]. URL disponible en: http://www.mailxmail.com/curso-etnociencias-yage/etnobiologiaintroduccion-paradigma-etnobiologia

Escobedo, A., C. Ríos. 2003. Uso de la fauna silvestre, peces y de otros productos forestales no maderables en las comunidades de las etnias Quechua y Achuar del río Huasaga, Loreto, Perú. Tesis para optar el título profesional de biólogo. Universidad Nacional de La Amazonía Peruana. Facultad de Ciencias Biológicas. 199 pp.

Guerrero, S., L. Ballesteros. 2006. Caracterización sociocultural y etnozoológica de la población asentada en la cabecera municipal de Quibdó, Chocó, Colombia. Trabajo de grado como requisito parcial para optar al título de trabajadora social. Quibdó: Universidad Tecnológica del Chocó. $116 \mathrm{pp}$.

Lugo, P. 2005. El manejo de la fauna silvestre asociada a las áreas de cultivo y su relación con elementos del sistema cultural en la comunidad Campesina de Vega Grande, Mogotes. Santander. Trabajo de grado para optar el título de ecólogo. Bogotá: Pontificia Universidad Javeriana. Facultad de Estudios Ambientales y Rurales, pp. 29-35.

Moreno, P., Z. Toral. 2001. Estudio biológico de la fauna de cacería (aves, reptiles y mamíferos) por parte de cazadores permanentes en el municipio de Cértegui, Chocó. Trabajo de grado. Quibdó: Universidad Tecnológica del Chocó. Facultad de Ciencias Básicas. Programa de Biología con Énfasis en Recursos Naturales. 61 pp.

Mosquera, J. 2001. Caracterización de la fauna de cacería (aves, mamíferos, reptiles y peces) en la comunidad de Buchadó Atrato Medio Antioquia. Trabajo de grado. Quibdó: Universidad Tecnológica del Chocó. Facultad de Ciencias Básicas. Programa de Biología con Énfasis en Recursos Naturales. 106 pp.

Moya, J. 2006. Composición y estructura de la comunidad de anuros presentes en la estación ambiental de Tutunendo, Chocó, Colombia. Trabajo de grado. Quibdó: Universidad Tecnológica del Chocó. Facultad de Ciencias Básicas. Programa de Biología con Énfasis en Recursos Naturales. $44 \mathrm{pp}$.

Ojasti, J. 1993. Utilización de la fauna silvestre en América Latina. Situación y perspectiva para un manejo sostenible. Roma: FAO. 248 pp.

Ojasti J. 2000. Manejo de fauna neotropical. En: F. Dallmeier (Ed). SIMAB. Serie $\mathrm{N}^{\circ}$ 5. Washington, DC: Smithsonian Institution/ MAB Program. 309 pp.

Páez, V., B. Bock, J. Estrada, A. Ortega, J. Daza, P. Gutiérrez. 2002. Guía de campo de algunas especies de anfibios y reptiles de Antioquia. Bogotá, Medellín: Universidad Nacional de Colombia, Conciencias, Universidad de Antioquia. pp. 137.

Poveda C., C. Rojas, A. Rudas, O. Rangel-Ch. 2004. Climas del Chocó Biogeográfico de Colombia. pp. 39-89. En: J. Rangel (Ed.). Colombia Diversidad Biótica IV. Chocó Biogeográfico/Costa Pacífica. Bogotá: Universidad Nacional de Colombia. Editorial Universidad Nacional de Colombia. 997 pp. 


\section{Bioetnia Volumen 9 № 2 (julio-diciembre), 2012}

Renjifo, J., M. Lundberg. 1999. Reptiles y anfibios de Urrá. Medellín: Ed. Colinas. 96 pp.

Rentería, L., J. Rengifo, J. Moya. 2007. Comunidad de reptiles presente en e sotobosque de la selva pluvial central del departamento del Chocó. Revista Institucional Universidad Tecnológica del Chocó: Investigación, Biodiversidad y Desarrollo 2007; 26 (2): 23-36.

Rentería L., T. Rivas. 2009. Etnozoología y caracterización taxonómica de la ofidio-fauna asociada a cultivos agrícolas presentes en la selva pluvial central del municipio de Quibdó, Chocó. En: Memorias del 1 Simposio de Herpetología en el Chocó Biogeográfico, Quibdó.

Rivas, T, A. Jiménez, J. Rengifo, J. Asprilla, Y. Moya. 2003. Fauna nativa del Chocó con perspectivas biomédicas. Revista Institucional Universidad Tecnológica del Chocó (19): 50-3.

Ulloa, A., H. Rubio, C. Campos. 1996. Trua Wuandra. Estrategias para el manejo de fauna con comunidades Emberá en el Parque Nacional Natural Ensenada de Utría, Chocó, Colombia. Bogotá: OREWA, Fundación Natura, UAESPNN, OEI. 